\title{
Genetic Stability, Active Constituent, and Pharmacoactivity of Salvia miltiorrhiza Hairy Roots and Wild Plant
}

\author{
Yuan Yuan ${ }^{\mathrm{a},}$, Yunjun Liu ${ }^{\mathrm{b}, \S}$, Dongmei Lu ${ }^{\mathrm{a}}$, Luqi Huang ${ }^{\mathrm{a}, *}$, Rixin Liang ${ }^{\mathrm{a}}$, \\ Zhaochun Yang ${ }^{\mathrm{a}}$, and Shunqin Chen ${ }^{\mathrm{a}}$ \\ a Institute of Chinese Materia Medica, Academy of Chinese Medical Sciences, \\ Beijing 100700, China. E-mail: huangluqi@263.net \\ b Institute of Crop Sciences, Chinese Academy of Agricultural Sciences, \\ Beijing 100081, China \\ * Author for correspondence and reprint requests \\ Z. Naturforsch. 64c, 557-563 (2009); received April 3/May 17, 2009
}

Salvia miltiorrhiza is an annual plant growing in China, Mongolia, Korea and some other Asian countries. The extract from $S$. miltiorrhiza roots has been used for supporting healthy cardiovascular and circulatory systems during the last decade. The active constituents of $S$. miltiorrhiza from different areas vary significantly, and the wild resources are overexploited. To adapt the demand for active constituents of S. miltiorrhiza against cardiovascular-related diseases, alternative materials need to be developed. The aim of the present work was to investigate the possibility of $S$. miltiorrhiza hairy roots as the alternative materials. The results showed that $S$. miltiorrhiza hairy roots are genetically stable. The contents of salvianolic acid B and tanshinone IIA, two main active constituents in hairy roots, determined by the assessment of combining flow cytometry and phytochemical analysis, are comparable to or significantly lower than in wild plant roots. The extract from $S$. miltiorrhiza hairy roots also had similar protection activity for hypoxia and reoxygenation injury in rat cardiac myocytes like that from wild plant roots. S. miltiorrhiza hairy roots may be alternative materials to obtain the drug or healthy food for cardiovascular-related diseases.

Key words: Salvia miltiorrhiza, Hairy Roots, Genetic Stability, Pharmacoactivity

\section{Introduction}

Salvia miltiorrhiza is an annual plant growing in China, Mongolia, Korea and some other Asian countries. S. miltiorrhiza root is an important herbal medicine and has been used for the treatment of cardiovascular-related diseases because it improves the myocardial energy metabolism level during prolonged hypoxia and subsequent reoxygenation, protects the myocardium against hypoxia/reoxygenation injury, significantly attenuates acidosis during hypoxia, and prevents the appearance of overacidic areas of the myocardium after reoxygenation (Lin et al., 2000). Because the active constituents of $S$. miltiorrhiza from different areas vary significantly and the wild resources

\footnotetext{
$\S$ These authors contributed equally to this work.

Abbreviations: DI, DNA fluorescence index; FCM, flow cytometry; MS, Murashige and Skoog; LDH, lactate dehydrogenase; MDA, malondialdehyde; SOD, superoxide dismutase; $\mathrm{H} / \mathrm{R}$, hypoxia and reoxygenation.
}

are overexploited, alternative materials need to be developed.

Hairy roots, obtained by infection with Agrobacterium rhizogenes, have a high growth rate and can grow in hormone-free media. Hairy root culture is an effective way to obtain active compounds of some medicinal plants, especially the interesting secondary metabolites that are accumulated in roots (Peebles et al., 2007). However, genetic instability would be induced during continuous in vitro maintenance. The percentage of Onobrychis viciaefolia hairy root cells with a normal chromosome number was reduced from $85.0 \%$ to $23.5 \%$ after four months of subculture, and only $4.1 \%$ left eight months later. A S. miltiorrhiza hairy root culture was established by infecting leaves with Agrobacterium rhizogenes strain 15834 (Zhang et al., 1995), and its mass culture was done in a 10-1 ball-type airlift bioreactor, obtaining $889.5 \mathrm{~g}$ fresh weight from $3.68 \mathrm{~g}$ inoculation mass and $1.1 \mathrm{mg} / \mathrm{g}$ dry weight tanshinone after 50 days. In a previous work in our laboratory, we studied the mechanism of active components 
accumulation in S. miltiorrhiza hairy roots. However, it is not clear whether the genetic change would occur during culture and how it affects the contents of active components.

In the present study, we assessed the genetic change and capacity to produce active constituents in S. miltiorrhiza hairy roots and roots of the wild plant, using an approach combining flow cytometry (FCM) and phytochemical analysis. We also evaluated the effects of the secondary metabolites from $S$. miltiorrhiza hairy roots on hypoxia and reoxygenation injury in rat cardiac myocytes. The results indicate that hairy roots may be a new source to obtain the drug against cardiovascular diseases, despite of the difference of nuclear DNA and active constituent contents to the wild plant.

\section{Material and Methods}

In vitro propagated hairy roots and phenotypic evaluation

S. miltiorrhiza is a diploid $(2 n=2 x=16)$ species. A $S$. miltiorrhiza hairy root culture was established by Zhang et al. (1995) and has been in vitro cultured on $67-\mathrm{V}$ medium till now. $1 \mathrm{~g}$ hairy roots was transferred to new solid $67-\mathrm{V}$ medium $(100 \mathrm{ml})$ and the experiment material was sampled every 6 weeks. 10 samples were taken and named as S1 to S10. One-year-old wild plants of $S$. miltiorrhiza were obtained from Xingtang planting base, Hebei, China and identified by Prof. Luqi Huang. Young shoots were sterilized with $0.1 \%$ $\mathrm{HgCl}_{2}$ and grown to seedlings on Murashige and Skoog (MS) medium. Root samples were used for FCM analysis 3 months later.

\section{Genomic PCR}

Genomic DNA was extracted from hairy root cultures and the wild plant as previously described. The rolC gene, an important gene in the T-DNA region of the $\mathrm{Ri}$ plasmid, was amplified using the PCR method to confirm the insertion of Ri T-DNA into the genome with the primers 5'-GATATATGCCAAATTTACACTAG-3' and 5'-GTTAACAAAGTAGGAAACAGG-3'. The expected PCR band size was $577 \mathrm{bp}$. The PCR conditions were as follows: $95^{\circ} \mathrm{C}$ for $1 \mathrm{~min}$, followed by 30 cycles at $94{ }^{\circ} \mathrm{C}$ for $1 \mathrm{~min}, 42^{\circ} \mathrm{C}$ for $1 \mathrm{~min}$, and $72{ }^{\circ} \mathrm{C}$ for $1 \mathrm{~min}$, with a final extension time of $10 \mathrm{~min}$ at $72{ }^{\circ} \mathrm{C}$.

\section{Quantitative analysis}

The contents of tanshinone IIA and salvianolic acid B in S. miltiorrhiza hairy roots and roots of the wild plant were analyzed according to the methods of Chinese Pharmacopoeia. Powdered material $(150 \mathrm{mg})$ obtained from samples was extracted for $1 \mathrm{~h}$ in a refluxing bath with $25 \mathrm{ml}$ methanol in the case of tanshinone IIA and 70\% methanol in the case of salvianolic acid B, respectively. The solutions were filtered through a membrane filter $(0.45 \mu \mathrm{m})$. Their concentrations were determined by a HPLC system with a flow rate of $1 \mathrm{ml} / \mathrm{min}$. HPLC conditions for the quantification of tanshinone IIA were as follows: Waters SymmetryShield ${ }^{\mathrm{TM}}$ (Milford, USA) $\mathrm{RP}_{18}$ column $(3.9 \mathrm{~mm} \times 150 \mathrm{~mm}, 5 \mu \mathrm{m})$, the elution solvent was $75 \%$ methanol and the detection wavelength was $270 \mathrm{~nm}$. HPLC conditions for the quantification of salvianolic acid B were: Waters SymmetryShield ${ }^{\mathrm{TM}} \mathrm{RP}_{18}$ column $(3.9 \mathrm{~mm} \times 150 \mathrm{~mm}, 5 \mu \mathrm{m})$, the elution solvent was $\mathrm{MeOH} / \mathrm{C}_{2} \mathrm{H}_{3} \mathrm{~N} / \mathrm{HCOOH} /$ $\mathrm{H}_{2} \mathrm{O}$ (30:10:1:59) and the detection wavelength was $286 \mathrm{~nm}$. Peaks were identified by comparison with the retention time of standards, which were purchased from National Institute for the Control of Pharmaceutical and Biological Products (China). Two standard solutions contained $8.56 \mu \mathrm{g} / \mathrm{ml}$ tanshinone IIA and $0.142 \mathrm{mg} / \mathrm{ml}$ salvianolic acid $\mathrm{B}$, respectively. The injection volume was $10 \mu \mathrm{l}$ for both standard solutions and sample solutions. The analyses were repeated 6 times.

\section{Flow cytometry analysis}

FCM analyses were carried out with S. miltiorrhiza hairy roots cultured on new medium for 6 weeks and 3-month-old wild plants cultured on MS medium. Glycine max (L.) Merrill was used as internal reference standard, the genome size of which has been captured recently as approx. $975 \mathrm{Mb}$ in 20 chromosomes (http://www.phytozome.net/soybean) and nuclear DNA (pg) was obtained as $1.99 \mathrm{pg} / 2 \mathrm{C}$ by converting $\mathrm{Mbp}$ to $\mathrm{pg}$ according to the factor $978 \mathrm{Mbp}=1 \mathrm{pg}$ (Dolezel et al., 2003). Nuclei samples were prepared according to a protocol adopted from Alan et al. (2007) with some modifications (Yuan et al., 2008). Briefly, $50 \mathrm{mg}$ of S. miltiorrhiza and the same amount of G. max leaf tissues were simultaneously chopped with a razor blade with $1 \mathrm{ml}$ of ice-cold nuclei isolation buffer [ $15 \mathrm{~mm}$ HEPES, $1 \mathrm{~mm} \mathrm{Na} \mathrm{NaDTA}_{2}$, $80 \mathrm{~mm} \mathrm{KCl}, 20 \mathrm{~mm} \mathrm{NaCl}, 300 \mathrm{~mm}$ sucrose, $0.2 \%$ 
(v/v) Triton X-100, $0.5 \mathrm{~mm}$ spermine, 1\% (w/v) polyvinylpyrrolidone-40, $\mathrm{pH}$ 7.5]. The homogenate was filtered into an 1.5-ml Eppendorf tube through a $37-\mu \mathrm{m}$ nylon filter and centrifuged at $8,000 \times g$ for $5 \mathrm{~min}$. The supernatant was poured out and the tube was placed upside down on a paper towel for several minutes to drain the remaining supernatant. The nuclei pellet was rinsed in $70 \%$ ethanol for $12 \mathrm{~h}$ and washed with isolation buffer for three times. The nuclei pellet was resuspended in $300 \mu \mathrm{l}$ isolation buffer with $25 \mu \mathrm{g} /$ $\mathrm{ml}$ RNase A. The nuclei were stained with $10 \mu \mathrm{l}$ of propidium iodide $(1 \mathrm{mg} / \mathrm{ml})$ per sample before analysis. The nuclei samples were also prepared without internal standard to determine the potential effect of G. max cell components on the DNA analysis. Samples were analyzed with a Coulter EPICS XL flow cytometer (Beckman-Coulter, Inc., Fullerton, CA, USA) equipped with an argon laser emitting at $488 \mathrm{~nm}$ using a $610-\mathrm{nm}$ band pass filter. The instrument was checked for linearity with fluorescent Flow Check beads (Beckman-Coulter) and the amplification was adjusted to position the $2 \mathrm{C}$ peak of hairy roots and wild plants of S. miltiorrhiza nuclei approx. at channel 120 and 360 separately. Ten nuclei samples were prepared and 3000-5000 nuclei were analyzed from each sample. The means and standard errors (SEs) of the nuclear DNA fluorescence in$\operatorname{dex}\left(\mathrm{DI}=2 \mathrm{C}_{\text {Sample }} / 2 \mathrm{C}_{\mathrm{G} \text {. max }}\right)$ and the nuclear DNA content ( $\mathrm{pg} / 2 \mathrm{C}$ ) were calculated according to the formula adopted from Dolezel and Bartos (2005). The mean coefficient variations $[\mathrm{CV}=(\mathrm{SD}$ of $2 \mathrm{C}$ peak/2C peak mean) $\cdot 100$ ] of $2 \mathrm{C}$ nuclei were calculated for all samples. Percentages of $2 \mathrm{C}, 4 \mathrm{C}$, and $8 \mathrm{C}$ nuclei were measured to detect the possible occurrence of mixoploidy and polyploidy. This was repeated three times.

\section{Evaluating the effects of secondary metabolites}

from S. miltiorrhiza hairy roots in hypoxia and reoxygenation injury on rat myocardial cells

Rat myocardial cells were cultured in Dulbecco's modified Eagle's medium (DMEM, Beijing Chemical Co, China) containing $20 \%$ fetal bovine serum (Hyclone Laboratories Inc., USA) for $3 \mathrm{~d}$. After $3 \mathrm{~h}$ of hypoxia and $1 \mathrm{~h}$ of reoxygenation, cells were incubated in different doses of $70 \%$ ethanolic extract from hairy roots (HP-1, HP-2, HP-3) and roots of the wild plant (WP-1, WP-2, WP-3). The beat frequency and cell survival were measured using an inverted microscope in hypoxia and reoxygenation. Then the cells were treated with $100 \mu \mathrm{l}$ 5'-diphenyl tetrazolium bromide solution (Sigma Chemical Co., USA, $0.5 \mathrm{mg} / \mathrm{ml}$, dissolved in DMEM without serum) at $37^{\circ} \mathrm{C}$ for $4 \mathrm{~h}$. After removing the solution, the cells were treated with $100 \mu$ l dimethyl sulfoxide (DMSO) and oscillated for $10 \mathrm{~min}$ to dissolve crystals sufficiently. The leakage of lactate dehydrogenase (LDH), content of malondialdehyde (MDA) and activity of superoxide dismutase (SOD) of upper solution were measured using ELISA kits purchased from Nanjing Jiancheng Bioengineering Institute, China. Data are analyzed by SPSS13.0 and presented as mean \pm SE.

\section{Results and Discussion}

Difference of nuclear DNA content between hairy roots and wild plants of $S$. miltiorrhiza

Our previous results have shown that the $S$. miltiorrhiza hairy root growth increased twelvefold after transferred to $67-\mathrm{V}$ medium for 6 weeks. After 6 weeks, hairy roots did not grow further and the contents of the active constituents tanshinone IIA and salvianolic acid B decreased. So, in the present study 6-week-old hairy roots were chosen as experiment material. To confirm the presence of the T-DNA fragment of Agrobacterium rhizogenes in hairy roots, PCR was performed with specific primers for the rolC gene, which should be inserted into genomic DNA to maintain the hairy root growth (Bonhomme et al., 2000). PCR results showed that the 577-bp rolC fragments were detected in all hairy root samples (Fig. 1), suggesting the stability of rolC and the T-DNA fragment of $A$. rhizogenes in long-term maintained $S$. miltiorrhiza hairy roots.

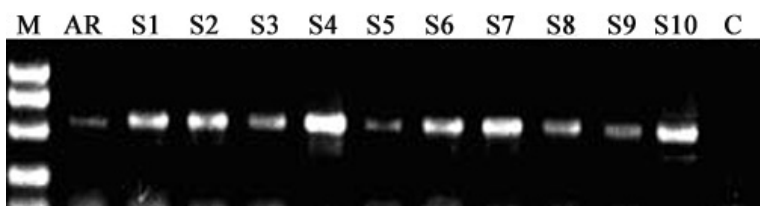

Fig. 1. PCR analysis of rolC gene in S. miltiorrhiza hairy roots. M, 2000-bp DNA marker; AR, Agrobacterium rhizogenes; S1-S10, different S. miltiorrhiza hairy root samples; C, wild plant of S. miltiorrhiza as control. Primers were designed specifically to obtain a 577-bp rolC gene fragment. 
Ploidy variation is easily induced in tissue cultures and can cause genetic instability. The nuclear DNA content can be well estimated by FCM in plants. Wu et al. (2006) reported the genetic variation of Chinese Cynodon using FCM analysis. In the present study, the possible variations in the nuclear DNA content and ploidy level of samples were detected by FCM analysis. First, we tried the nuclei sample preparation protocol from Alan et al. (2007); however, it was not suitable for the hairy roots because of the big noise in FCM analysis. So we modified the method using root tissue as sample. The modified method was successfully used in FCM analyses of the Scutellaria baicalensis genome size using root samples (data not shown), which is similar to the results using leaf samples (Alan et al., 2007). For FCM analyses of S. miltiorrhiza hairy roots and wild plants, only those samples with 3000-5000 nuclei were used. The nuclei samples of S. miltiorrhiza hairy roots and wild plants, separately, showed sharp 2C peaks with CVs averaging $6.7 \%$ and $6.9 \%$, and 4C peaks were barely detectable above the debris. The ranges of CVs in the present study were similar to the analysis results from Scutellaria baicalensis (Alan et al., 2007). The results of cytogenetic analysis showed that the frequency of chromosomic alterations was very low in hairy roots of Datura stramonium, which is not the case with normal root cultures (Baiza et al., 1999). G. max nuclei were used as internal standard and did not change the positions of $S$. miltiorrhiza hairy root and wild plant peaks. FCM analysis of S. miltiorrhiza hairy roots provided mean nuclear DIs of 0.197 , ranging from 0.193 to 0.225 , and nuclear DNA contents ranging from 0.384 to $0.448 \mathrm{pg} / 2 \mathrm{C}$. Our results showed that the diploid level was well preserved among in vitro maintained hairy roots, with nuclei rate in $2 \mathrm{C}$ peaks. Nuclear DNA contents were similar among the 10 samples of hairy roots, confirming the genetic stability of in vitro long-term cultured hairy roots. While Walker et al. (2006) reported that environmental factor effects on DNA determination of Fabaceae populations and the mean nuclear DNA content correlated significantly with the mean temperature and longitude, in our study, wild plants provided mean nuclear DIs of 0.599 and nuclear DNA contents of $1.192 \mathrm{pg} / 2 \mathrm{C}$. The $1 \mathrm{C}$ genome size of hairy roots decreased compared with the wild plant. A genetic change was found between hairy roots and the wild plant of $S$. miltiorrhiza, suggesting that plants may discard their unnecessary nuclear DNA when they only have a root organ.

\section{Comparison of active constituents in hairy roots and wild plants of $S$. miltiorrhiza}

Contents of tanshinone IIA and salvianolic acid B, the important indices for the quality of $S$. miltiorrhiza medicine, were determined in methanolic extracts of hairy roots. In a typical HPLC chromatogram (Fig. 2), peaks 1 and 2 were readily identified as tanshinone IIA and salvianolic acid
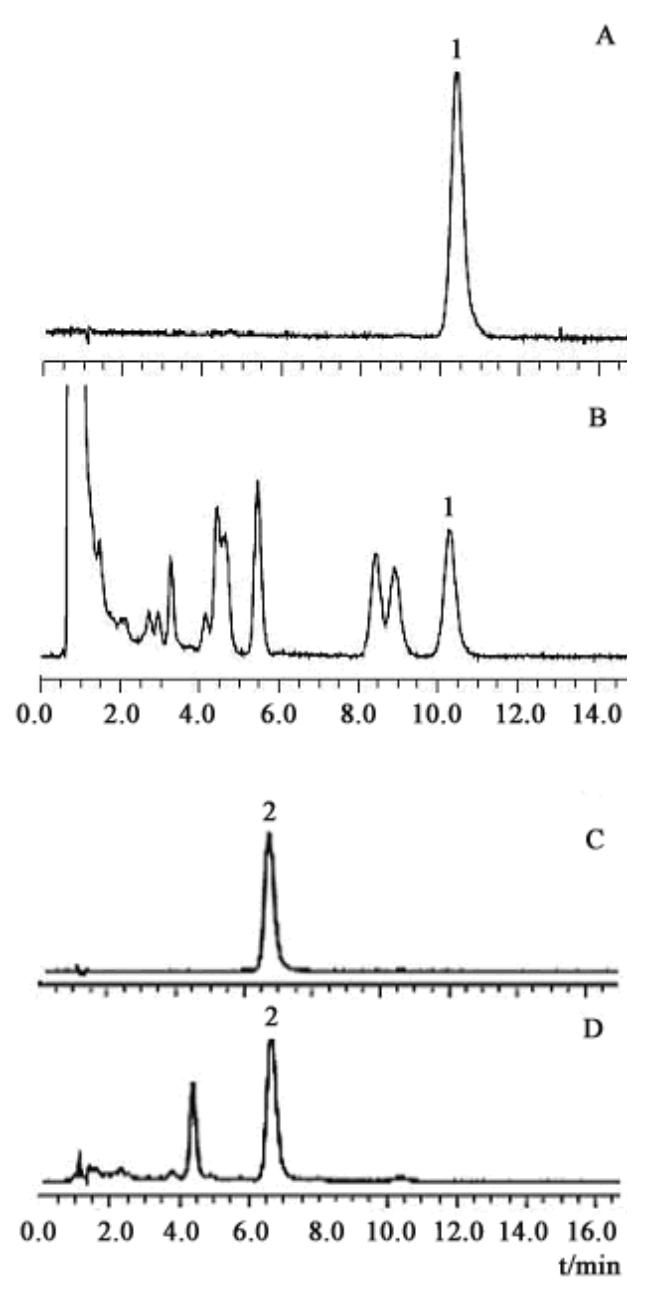

Fig. 2. HPLC analysis of S. miltiorrhiza hairy roots and wild plants. (A) Standard sample of tanshinone IIA; (B) mean chromatogram of $S$. miltiorrhiza hairy root samples for detecting tanshinone IIA; (C) standard sample of salvianolic acid B; (D) mean chromatogram of $S$. miltiorrhiza hairy root samples for detecting salvianolic acid $\mathrm{B} ; 1$, tanshinone IIA; 2 , salvianolic acid $\mathrm{B}$. 

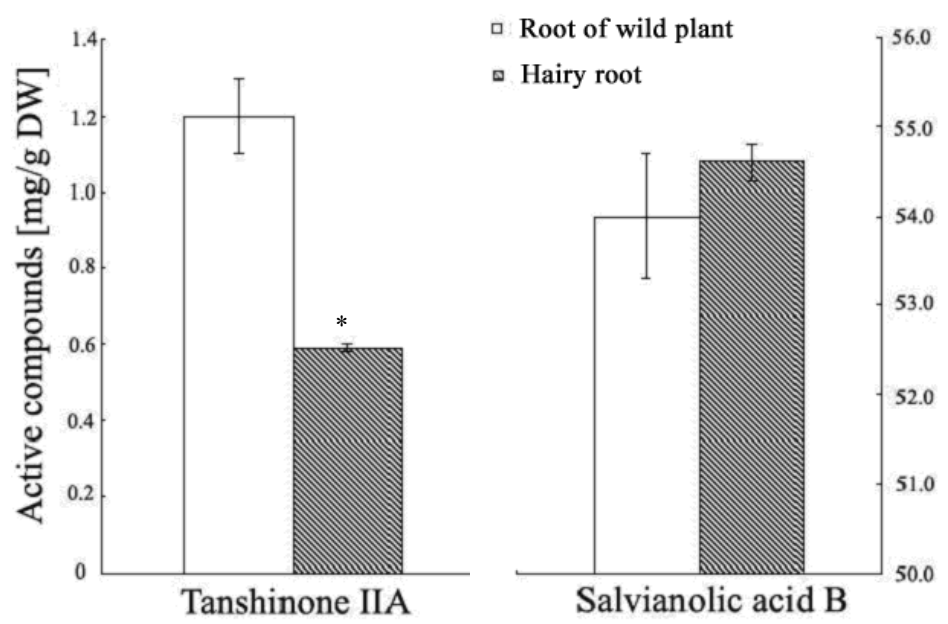

Fig. 3. Contents of tanshinone IIA and salvianolic acid B in S. miltiorrhiza hairy roots and roots of wild plant samples. Data are shown as means \pm SE for 6 independent experiments; * denotes the significant difference between hairy roots and roots of wild plants at $P<0.05$.

B, respectively, by comparing their UV spectra and retention times with those of corresponding authentic standards. The results showed that all $S$. miltiorrhiza hairy root samples maintained the capacity to produce characteristic active constituents under in vitro conditions. On average, the hairy root samples contained $0.59 \mathrm{mg} / \mathrm{g}$ tanshinone IIA and $54.6 \mathrm{mg} / \mathrm{g}$ salvianolic acid B (Fig. 3). But significant differences were demonstrated in these two substances amounts in in vitro maintained hairy roots. The CVs of tanshinone IIA and salvianolic acid B content in all hairy root samples was $18.9 \%$ and $21.1 \%$, respectively, which was lower than the $50.0 \%$ and $25.3 \%$ of five cultivated populations (Zeng et al., 2006), suggesting the quality stability of hairy roots as medicine. Fu et al. (2006) reported that the genetic modification of roots leads to differential regulation mechanisms of gene expression for even closely related compounds, causing different contents of active constituents between hairy roots and wild plants. Hairy root cultures in this study did not, however, exhibit superior potentials for active compounds. The contents of tanshinone IIA and salvianolic acid B in hairy root cultures were about half of or similar to those in the roots of wild plants. The contents of active constituents in hairy roots could be regulated easier than in wild plant roots, so $S$. miltiorrhiza hairy roots might be a better material to obtain active constituents, $e$. $g$. tanshinone IIA and salvianolic acid $\mathrm{B}$, than plant roots.

Table I. Effect in beat frequency of rat myocardial cells in hypoxia/reoxygenation injury.

\begin{tabular}{|c|c|c|c|c|c|}
\hline Parameter/group & & $\begin{array}{c}\text { Tanshinone IIA } \\
{[\mu \mathrm{g} / \mathrm{ml}]}\end{array}$ & $\begin{array}{c}\text { Salvianolic acid B } \\
{[\mu \mathrm{g} / \mathrm{ml}]}\end{array}$ & $\begin{array}{c}\text { Hypoxia } \\
{\left[\mathrm{min}^{-1}\right]}\end{array}$ & $\begin{array}{c}\text { Reoxygenation } \\
{\left[\mathrm{min}^{-1}\right]}\end{array}$ \\
\hline Control & & - & - & $69 \pm 10$ & $67 \pm 6^{*}$ \\
\hline $\mathrm{H} / \mathrm{R}$ & & - & - & $69 \pm 16$ & $38 \pm 7$ \\
\hline \multirow[t]{3}{*}{ Roots of wild plants } & WP-1 & 0.35 & 15.66 & $70 \pm 14$ & $54 \pm 7 * *$ \\
\hline & WP-2 & 0.09 & 3.94 & $66 \pm 14$ & $46 \pm 3 *$ \\
\hline & WP-3 & 0.02 & 0.97 & $70 \pm 19$ & $38 \pm 5$ \\
\hline \multirow[t]{3}{*}{ Hairy roots } & HR-1 & 0.35 & 15.66 & $71 \pm 8$ & $58 \pm 11 * *$ \\
\hline & HR-2 & 0.09 & 3.94 & $70 \pm 14$ & $50 \pm 7 *$ \\
\hline & HR-3 & 0.02 & 0.97 & $69 \pm 11$ & $48 \pm 11$ \\
\hline
\end{tabular}

* and ** denote the significant difference to H/R group at $P<0.05$ and $P<0.01$ level. 
Table II. Cell viability, LDH, MDA, and SOD of rat myocardial cells in hypoxia/reoxygenation injury.

\begin{tabular}{|c|c|c|c|c|c|c|c|}
\hline \multicolumn{2}{|c|}{ Parameter/group } & $\begin{array}{l}\text { Tanshinone } \\
\text { IIA }[\mu \mathrm{g} / \mathrm{ml}]\end{array}$ & $\begin{array}{c}\text { Salvianolic } \\
\text { acid B }[\mu \mathrm{g} / \mathrm{ml}]\end{array}$ & $\begin{array}{c}\begin{array}{c}\text { Cell viability } \\
(\%)\end{array} \\
\end{array}$ & $\begin{array}{l}\text { LDH } \\
{[\mathrm{U} / \mathrm{l}]} \\
\end{array}$ & $\begin{array}{c}\mathrm{MDA} \\
{[\mathrm{nmol} / \mathrm{l}]} \\
\end{array}$ & $\begin{array}{l}\text { SOD } \\
{[\mathrm{U} / 1]}\end{array}$ \\
\hline Control & & - & - & $79.9 \pm 2.4 * * *$ & $229.6 \pm 101.1 * * *$ & $1.4 \pm 0.1^{*}$ & $3.0 \pm 0.7 * *$ \\
\hline$H / R$ & & - & - & $67.5 \pm 1.7$ & $1179.0 \pm 52.0$ & $1.7 \pm 0.2$ & $1.8 \pm 0.8$ \\
\hline \multirow{3}{*}{$\begin{array}{l}\text { Roots of wild } \\
\text { plants }\end{array}$} & WP-1 & 0.35 & 15.66 & $72.7 \pm 3.8^{*}$ & $946.7 \pm 67.7 * *$ & $0.9 \pm 0.2^{* * *}$ & $3.8 \pm 0.2 * * *$ \\
\hline & WP-2 & 0.09 & 3.94 & $71.1 \pm 1.1$ & $1042.8 \pm 100.0^{*}$ & $1.1 \pm 0.2^{* * *}$ & $4.1 \pm 0.5^{* * *}$ \\
\hline & WP-3 & 0.02 & 0.97 & $71.3 \pm 1.5$ & $1068.5 \pm 142.3$ & $1.2 \pm 0.2 * *$ & $4.3 \pm 0.5 * * *$ \\
\hline \multirow[t]{3}{*}{ Hairy roots } & HR-1 & 0.35 & 15.66 & $70.1 \pm 1.7 * *$ & $1142.9 \pm 85.2$ & $1.1 \pm 0.1^{* * *}$ & $4.3 \pm 0.1 * * *$ \\
\hline & HR-2 & 0.09 & 3.94 & $69.5 \pm 1.8^{* *}$ & $1169.2 \pm 91.8$ & $1.2 \pm 0.2 * *$ & $3.9 \pm 0.4 * * *$ \\
\hline & HR-3 & 0.02 & 0.97 & $67.7 \pm 0.1 * *$ & $1177.7 \pm 61.0$ & $1.5 \pm 0.6$ & $3.6 \pm 1.1 * * *$ \\
\hline
\end{tabular}

*, ** and $* * *$ denote the significant difference to $\mathrm{H} / \mathrm{R}$ group at $P<0.05, P<0.01$ and $P<0.001$ level.

Protective effects of $S$. miltiorrhiza hairy roots against myocardial hypoxia/reoxygenation injury

It has been reported that the medicinal function of S. miltiorrhiza is to cure cardiovascular disease (Wei et al., 2007). Our results showed that the content of the active compound tanshinone IIA in hairy roots is lower than that in plant roots, causing the question whether S. miltiorrhiza hairy roots could maintain the pharmcoactivity. Thus pharmalogical experiments using the extract from S. miltiorrhiza hairy roots against myocardial hypoxia/reoxygenation $(H / R)$ injury in rat myocardial cells were performed.

Our results showed that the rat myocardial cells were injured significantly in the $H / R$ group. The beat frequency of injured cells increased significantly after rat myocardial cells were treated with different doses of the extract from hairy roots and roots of wild plants (Table I). Compared with the control group, the absorbency decreased in the methylthiazolyldiphenyl tetrazolium experiment. The leakage of LDH and content of MDA in culture solution increased, but the activity of SOD decreased. Compared with the H/R group, the number of live cells increased after being treated with the extract from hairy roots (HR-1, HR-2,

Alan A. R., Zeng H., Assani A., Shi W. L., McRae H. E., and Murch S. J. (2007), Assessment of genetic stability of the germplasm lines of medicinal plant Scutellaria baicalensis Georgi (Huang-qin) in long-term, in vitro maintained cultures. Plant Cell Rep. 26, 1345-1355.

Baiza A., Quiroz-Moreno A., Ruiz J., and Loyola-Vargas V. (1999), Genetic stability of hairy root cultures
HR-3) and in the WP-1 group. There was no significant difference in the LDH release between the wild plant groups and hairy root groups. The MDA level in culture solution decreased in the three wild plant groups, HR-1 group, and HR-2 group, and the activity of SOD increased in the three wild plant groups and the three hairy root groups. The LDH and MDA level decreased following the increase of drug dosage (Table II). These results suggest that $S$. miltiorrhiza hairy roots could resist injury in rat cardiac myocytes and prevent rat cardiac myocytes from $\mathrm{H} / \mathrm{R}$ injury, the mechanism of which is likely related to antioxidation and scavenging of free radicals from cardiomycytes. The results show that the extraction from $S$. miltiorrhiza hairy roots has similar protection activity to that from wild plant roots, suggesting that $S$. miltiorrhiza hairy roots might be used as alternative to $S$. miltiorrhiza roots.

\section{Acknowledgements}

This work was supported by the China National Basic Research Program (grant No. 2006CB504700) and by Chinese National Programs for High Technology Research and Development (grant No. 2007AA02Z104). of Datura stramonium. Plant Cell Tiss. Org. Cult. 59, 9-17.

Bonhomme V., Laurain-Mattar D., and Fliniaux M. A. (2000), Effects of the rol C gene on hairy root: induction development and tropane alkaloid production by Atropa belladonna. J. Nat. Prod. 63, 1249-1252.

Dolezel J. and Bartos J. (2005), Plant DNA flow cytometry and estimation of nuclear genome size. Ann. Bot. (London) 95, 99-110. 
Dolezel J., Bartos J., Voglmayr H., and Greilhuber J. (2003), Nuclear DNA content and genome size of trout and human. Cytometry A 51, 127-128.

Fu C. X., Xu Y. J., Zhao D. X., and Ma F. S. (2006), A comparison between hairy root cultures and wild plants of Saussurea involucrata in phenylpropanoids production. Plant Cell Rep. 24, 750-754.

Lin L. Z., Wu C. L., Lu Z., Huang Y. R., Zhuang J. G., and Zhou Z. N. (2000), The studies on protective effects of SM against myocardial hypoxia/reoxygenation injury. Zhongguo Ying Yong Sheng Li Xue Za Zhi 16, 324-326.

Peebles C. A., Gibson S. I., Shanks J. V., and San K. Y. (2007), Long-term maintenance of a transgenic $\mathrm{Ca}$ tharanthus roseus hairy root line. Biotechnol. Prog. 23, $1517-1518$.

Walker D. J., Monino I., and Correal E. (2006), Genome size in Bituminaria bituminosa (L.) C. H. Stirton (Fabaceae) populations: separation of "true" differences from environmental effects on DNA determination. Environ. Exp. Bot. 55, 258-265.
Wei L., Jianhua L., Mala A., Rongqian W., Dazhi C., and Lihong Y. (2007), A cardiovascular drug rescues mice from lethal sepsis by selectively attenuating a late-acting proinflammatory mediator, high mobility group box 1. J. Immunol. 178, 3856-3864.

Wu Y. Q., Taliaferro C. M., Bai G. H., Martin D. L., Anderson J. A., and Anderson M. P. (2006), Genetic analyses of Chinese cynodon accessions by flow cytometry and AFLP markers. Crop Sci. 46, 917-926.

Yuan Y., Yang Z. C., Lu D. M., Chen M., and Huang L. Q. (2008), Measurement of Salvia miltiorrhiza hairy root nuclear DNA content with flow cytometry method. Chin. J. Exp. Trad. Med. Form. 12, 24-25.

Zeng L., Liang H., Chen Y., Liang H., and Pan Y. (2006), Primary study on the distribution of tanshinone IIA and salvianolic acid B in radix Salvia miltiorrhiza. Res. Pract. Chin. Med. 20, 7-9.

Zhang Y. L., Song J. Y., Liu G. L., and Liu H. L., (1995), Establishment of hairy root culture and production of tanshinone in Salvia miltiorrhiza, China. J. Chin. Material Med. 20, 269-271. 